\title{
Timed Up and Go teste na avaliação do risco de quedas em idosos: uma revisão de literatura
}

\author{
Timed Up and Go test in the assessment of the risk of falls in the elderly: a literature review \\ Prueba Timed Up and Go en la evaluación del riesgo de caídas en ancianos: revisión de la literatura
}

Recebido: 06/10/2021 | Revisado: 12/10/2021 | Aceito: 12/10/2021 | Publicado: 15/10/2021

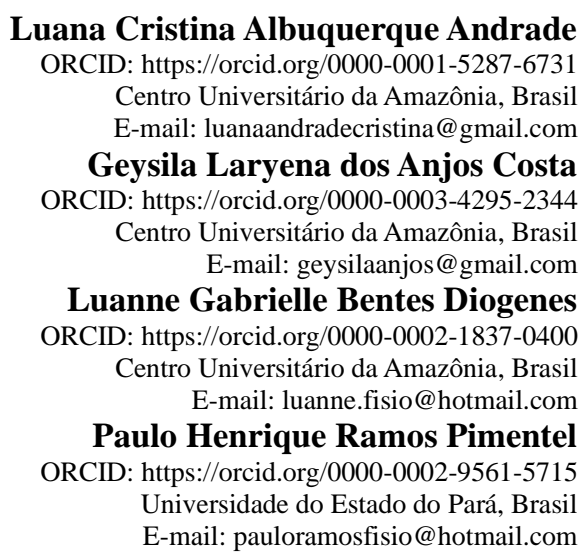

\begin{abstract}
Resumo
Avaliar o risco de quedas em idosos é de conhecimento fundamental para a criação de políticas públicas de atenção à saúde. O objetivo do trabalho foi investigar a utilização do teste Timed Up and Go (TUG) como ferramenta na avaliação do risco de quedas em idosos. Trata-se de uma revisão de literatura de artigos científicos indexados nas bases de dados PubMed e Scielo, publicados entre 2011 e 2021. Com isso os resultados obtidos nos estudos não conseguiram demostrar a eficiência do TUG para avaliar o risco de quedas em idosos, além das divergências analisadas, principalmente em relação a nota de corte, pois existem trabalhos que apontam medidas diferentes. Os estudos não conseguiram demostrar a eficácia do TUG na predição de quedas em idosos, mas demostraram importância quando utilizado com outros testes. Observa-se a necessidade de mais estudos sobre a temática, pois podem contribuir para a prática clínica, orientando a melhor escolha de testes a serem aplicados em idosos que apresentam risco de quedas.
\end{abstract}

Palavras-chave: Acidentes por quedas; Avaliação geriátrica; Equilíbrio postural.

\begin{abstract}
Assess the risk of falls in the elderly and fundamental knowledge for the creation of public health care policies. The aim of this study was to investigate the use of the Timed Up and Go (TUG) test as a tool to assess the risk of falls in the elderly. This is a literature review of scientific articles indexed in the PubMed and Scielo databases, published between 2011 and 2021. Thus, the results obtained in the studies failed to demonstrate the efficiency of the TUG to assess the risk of falls in the elderly, in addition to the divergences analyzed, especially in relation to the cutoff score, as there are studies that point to different measures. Studies have failed to demonstrate the effectiveness of TUG in predicting falls in the elderly, but demonstrate its importance when used with other tests. There is a need for more studies on the subject, as they can contribute to clinical practice, guiding the best choice of tests to be applied to elderly people who are at risk of falls.
\end{abstract}

Keywords: Accidental falls; Geriatric assessment; Postural balance.

\section{Resumen}

Valorar el riesgo de caídas en las personas mayores y conocimientos fundamentales para la creación de políticas públicas de salud. El objetivo de este estudio fue investigar el uso del test Timed Up and Go (TUG) como herramienta para evaluar el riesgo de caídas en ancianos. Se trata de una revisión de la literatura de artículos científicos indexados en las bases de datos PubMed y Scielo, publicados entre 2011 y 2021. Por tanto, los resultados obtenidos en los estudios no lograron demostrar la eficiencia del TUG para evaluar el riesgo de caídas en el anciano, además de las divergencias analizadas, especialmente en relación al puntaje de corte, ya que existen estudios que apuntan a diferentes medidas. Los estudios no han podido demostrar la eficacia de TUG para predecir caídas en los ancianos, pero demuestran su importancia cuando se usa con otras pruebas.Se necesitan más estudios sobre el tema, ya que 
pueden contribuir a la práctica clínica, orientando la mejor elección de pruebas a aplicar a las personas mayores con riesgo de caídas.

Palabras clave: Accidentes por caídas, Évaluación gériátrica; Equilibrio postural.

\section{Introdução}

O processo de envelhecimento acontece no decorrer da vida, de forma gradativa, levando a inúmeras transformações no organismo do sujeito, podendo abranger diversos fatores, tais como: fatores culturais, sociais, biológicos e psicológicos (Cunha et al., 2019). A população com mais de 60 anos no Brasil em 2000, era de 14,5 milhões de pessoas. Um aumento considerável de 35,5\% comparado ao ano de 1991. Em 2019 a população idosa era de 29 milhões e a probabilidade é de que até 2060 essa população alcance um total de 73 milhões, evidenciando um crescimento de 160\% (Lima-costa, 2018; Sociedade Brasileira de Geriatria e Gerontologia, 2019).

Algumas modificações fisiológicas e funcionais ocorrem durante o processo de envelhecimento, tais como: diminuição da capacidade visual, redução do equilíbrio e força muscular, elevando o risco de quedas e possíveis lesões decorrentes delas (Gamage, et al., 2019, Rodrigues et al., 2014, Teixeira et al., 2019).

O episódio de queda em idosos é uma das maiores consequências do envelhecimento. Pesquisas têm registrado diferentes fatores de risco que justificam tal acontecimento, por exemplo: o avanço da idade, sexo feminino, fraqueza muscular, instabilidade na marcha, equilíbrio alterado, déficit cognitivo, e sintomas de depressão- (Fang et al., 2012; Jagnoor et al.,2014). Sabe-se que a queda é conceituada como um episódio não proposital, onde o corpo se desloca para uma altura abaixo da posição inicial- (Teresi, et al., 2013; Baixinho et al., 2019). Por esse motivo, este é o acidente mais comum que acomete os idosos, gerando consequências, tais como: redução da qualidade de vida, provocando medo de um novo episódio de queda, isolamento social e desenvolvendo limitações funcionas (Damián, et al. 2013).

Além disso, a queda está relacionada a um conjunto de fatores, tornando crucial o conhecimento multidisciplinar com intuito de proporcionar informações a respeito da prevenção e identificação dos idosos que correm tal risco. Para que medidas preventivas sejam firmadas, é fundamental verificar esses fatores associados à queda, com a intenção de reparar os fatores alteráveis, diminuindo assim a incidência das mesmas. (Smith, et al., 2017).

Perante estes acontecimentos, o teste Timed Up and Go (TUG), tem o objetivo de analisar a mobilidade e o equilíbrio funcional sobretudo entre adultos mais velhos (Podsiadlo \& Richardson, 1991). O TUG é um teste de simples execução, sem falar no custo e na eficácia de avaliação da mobilidade e do equilíbrio funcional, além disso vem sendo bastante procurado por se associar ao risco de quedas, o medo de cair e sua funcionalidade (Alexandre, et al., 2012; Thrane, et al., 2007).

O TUG consiste na ação do indivíduo de levantar de uma cadeira e se deslocar em uma distância de 3 metros à frente, virar-se, deambular de volta e sentar na cadeira novamente (Podsiadlo \& Richardson, 1991).

Apesar de este ser um teste muito utilizado, há discordância quanto aos valores de referência para definir se idosos estão ou não sujeitos a quedas (Rodrigues, Souza, 2016). Segundo Bischoff, et al., (2003) resultados apontados como normal para o TUG, é quando o sujeito leva um tempo de até 10 segundos para realizar o teste. Quando o resultado atinge 11-20 segundos, considera-se que o indivíduo seja parcialmente independente e possua baixo risco de quedas. Já quando se obtém o resultado superior a 20 segundos, o sujeito demonstra déficit significativo da mobilidade física e alto risco de quedas. Diante do exposto, o presente estudo tem como objetivo principal investigar a utilização do teste Timed Up And Go na avaliação do risco de quedas em idosos. 


\section{Metodologia}

Trata-se de uma revisão de literatura exploratória descritiva, com estratégia qualitativa, que é realizada de forma detalhada do tema estudado com intuito de abranger mais sobre determinada temática. (Rother, 2007).

Para a construção dessa pesquisa foram utilizados os seguintes passos: determinar o tema; criação de critérios de inclusão e exclusão; seleção dos estudos; analise e a averiguação dos resultados. Para direção da elaboração dessa revisão norteou como questão problema: "O teste Timed Up and Go pode ser utilizado para avaliar equilíbrio e predizer o risco de quedas em idosos?"

As bases de dados utilizadas foram Pub Med e Scielo. Nas plataformas Pubmed e Scielo foram pesquisadas as palavras chave: Timed Up and Go; Acidentes por Quedas; Avaliação geriátrica; equilíbrio postural. Correspondentes na língua inglesa: Timed Up and Go; Acidental Falls; Geriatric Assessment; postural balance.

Os critérios de inclusão foram: Estudos publicados nos últimos 10 anos (2011-2021) com coerência do tema proposto na língua inglesa e portuguesa.

Os critérios de exclusão: estudos que não se adequavam com o tema, estudos de casos, publicações anteriores ao ano de 2011.

Após a análise detalhada dos artigos que se contemplavam os filtros das pesquisas. Foram utilizados 10 artigos que dirigia o tema pesquisado.

\section{Resultados}

No Quadro 1 mostra-se 10 estudos realizados em idosos de ambos os sexos que foram submetidos a avaliação de equilíbrio através do teste TUG.

Quadro 1 - Tabela com os resultados de estudos publicados nos anos de 2011 a 2021.

\begin{tabular}{|c|c|c|c|c|}
\hline Autores (Ano) & Objetivo & Amostra & Intervenção & Conclusão \\
\hline $\begin{array}{l}\text { BRETAN O, et al, } \\
2013 \text {. }\end{array}$ & $\begin{array}{l}\text { Avaliar a utilização do } \\
\text { teste TUG para } \\
\text { mensurar o equilíbrio } \\
\text { dos idosos. }\end{array}$ & $\begin{array}{l}\text { Realizado com } 102 \\
\text { idosos sendo } 66 \% \text { do } \\
\text { sexo feminino e } 34 \% \\
\text { do sexo masculino, } \\
\text { com idade igual ou } \\
\text { superior a } 75 \text { anos } \\
\text { ou mais. }\end{array}$ & $\begin{array}{l}\text { Este estudo realizou uma pesquisa } \\
\text { de história clínica dos pacientes } \\
\text { para obter dados sobre as doenças } \\
\text { diagnosticadas pelo clínico, que } \\
\text { possam favorecer queda. Além } \\
\text { disso, utilizaram o TUG para a } \\
\text { avaliação de equilíbrio dos idosos } \\
\text { envolvidos. }\end{array}$ & $\begin{array}{l}\text { Com os resultados da pesquisa } \\
\text { pode-se observar que uma boa } \\
\text { parte dos idosos demostraram boa } \\
\text { mobilidade funcional, mas um } \\
\text { determinado número de idosos } \\
\text { demostraram altos valores no } \\
\text { teste e são mais propensos a } \\
\text { quedas. }\end{array}$ \\
\hline $\begin{array}{l}\text { VIRTUOSO J. F, et al, } \\
2013 \text {. }\end{array}$ & $\begin{array}{l}\text { Analisar a versão } \\
\text { clássica e cognitiva do } \\
\text { TUG, na previsão e } \\
\text { explicação de episódio } \\
\text { de quedas, em idosos } \\
\text { que praticam exercício } \\
\text { físico. }\end{array}$ & $\begin{array}{l}\text { Participaram } 82 \\
\text { idosos fisicamente } \\
\text { ativos de ambos os } \\
\text { sexos. }\end{array}$ & $\begin{array}{l}\text { Realizou-se o teste TUG clássico e } \\
\text { o cognitivo por meio da pronúncia } \\
\text { de animas enquanto o idoso } \\
\text { executava o teste. }\end{array}$ & $\begin{array}{l}\text { O estudo demostrou que testes } \\
\text { TUG clássico e cognitivo não se } \\
\text { associam com a ocorrência de } \\
\text { quedas e deve se ter um cuidado } \\
\text { ao utilizar na previsão de quedas } \\
\text { em idosos que praticam exercício } \\
\text { física. }\end{array}$ \\
\hline $\begin{array}{l}\text { CAMPOS, M.P.S, et al, } \\
2013 .\end{array}$ & $\begin{array}{l}\text { Identificar os fatores } \\
\text { de risco de quedas em } \\
\text { idosos e analisar se os } \\
\text { testes de equilíbrio, } \\
\text { TAF e TUG, } \\
\text { identificam indivíduos } \\
\text { com maior } \\
\text { possibilidade de cair. }\end{array}$ & $\begin{array}{l}\text { A pesquisa foi } \\
\text { realizada com } 155 \\
\text { idosos, contendo } 131 \\
\text { mulheres e } 24 \\
\text { homens. }\end{array}$ & $\begin{array}{l}\text { Este estudo utilizou dois testes } \\
\text { funcionais de equilíbrio: TAF e } \\
\text { TUG, no período de abril a } \\
\text { novembro de } 2007 \text {. }\end{array}$ & $\begin{array}{l}\text { O estudo concluiu que os dois } \\
\text { testes não conseguiram identificar } \\
\text { idosos com probabilidade a } \\
\text { quedas. }\end{array}$ \\
\hline $\begin{array}{l}\text { KOJIMA, G, et al, } \\
2015 .\end{array}$ & $\begin{array}{l}\text { Examinar a } \\
\text { capacidade do TUG de } \\
\text { prever futuras quedas } \\
\text { e estimar o ponto de } \\
\text { corte ideal para definir } \\
\text { aqueles com maior } \\
\text { risco de quedas. }\end{array}$ & $\begin{array}{l}\text { Pesquisa realizada } \\
\text { com } 259 \text { idosos com } \\
\text { idade igual ou } \\
\text { superior à } 65 \text { anos. }\end{array}$ & $\begin{array}{l}\text { Este estudo realizou o TUG com os } \\
\text { participantes, onde foram } \\
\text { observados e cronometrados. As } \\
\text { quedas foram monitoradas ao longo } \\
\text { de } 24 \text { semanas por meio de um } \\
\text { diário de quedas enviados a cada } 4 \\
\text { semanas, totalizando seis diários. }\end{array}$ & $\begin{array}{l}\text { Os resultados encontrados } \\
\text { demostraram que a capacidade do } \\
\text { TUG para prever quedas é } \\
\text { limitado, mas identificou que o } \\
\text { teste pode ser utilizado em casos } \\
\text { de alto risco de quedas. }\end{array}$ \\
\hline
\end{tabular}




\begin{tabular}{|c|c|c|c|c|}
\hline $\begin{array}{l}\text { RODRIGUES A.L.P; } \\
\text { SOUZA V. R, } 2016 .\end{array}$ & $\begin{array}{l}\text { Verificar a eficiência } \\
\text { do teste TUG na } \\
\text { predição de quedas em } \\
\text { idosos atendidos em } \\
\text { uma unidade básica de } \\
\text { saúde em Fortaleza- } \\
\text { CE. }\end{array}$ & $\begin{array}{l}\text { Realizado com } 55 \\
\text { idosos de ambos os } \\
\text { sexos, com idade } \\
\text { acima de } 60 \text { anos. }\end{array}$ & $\begin{array}{l}\text { Este estudo utilizou um questionário } \\
\text { sócio demográfico, também realizou } \\
\text { o teste TUG para avaliar a } \\
\text { capacidade físico-funcional } \\
\text { relacionados ao equilíbrio e ao risco } \\
\text { de quedas com cada paciente. }\end{array}$ & $\begin{array}{l}\text { Pôde concluir que o teste se } \\
\text { mostrou eficaz na predição de } \\
\text { ocorrência de quedas nos idosos. }\end{array}$ \\
\hline $\begin{array}{l}\text { MARTINEZ, B. P, et al, } \\
2016 .\end{array}$ & $\begin{array}{l}\text { Avaliar a segurança e } \\
\text { reprodutibilidade do } \\
\text { TUG em idosos } \\
\text { internados em um } \\
\text { hospital em Salvador- } \\
\text { BA. }\end{array}$ & $\begin{array}{l}\text { Foi realizado com } 68 \\
\text { idosos } \\
\text { hospitalizados com } \\
\text { idade igual ou maior } \\
\text { que } 60 \text { anos, } \\
\text { internados entre o } 1^{\circ} \\
\text { e } 5^{\circ} \text { dia de } \\
\text { hospitalização. }\end{array}$ & $\begin{array}{l}\text { Este estudo utilizou o TUG para } \\
\text { avaliação do desempenho físico no } \\
\text { período de agosto de } 2013 \text { a janeiro } \\
\text { de } 2014 \text {. Foram executadas no total } \\
3 \text { aferições em cada idoso com } \\
\text { intervalo de } 1 \text { min entre as medidas, } \\
\text { sendo considerado melhor } \\
\text { desempenho como resultado final }\end{array}$ & $\begin{array}{l}\text { Conclui-se que o teste TUG é um } \\
\text { instrumento seguro e de boa } \\
\text { reprodutibilidade para avaliar o } \\
\text { desempenho físico em idosos. }\end{array}$ \\
\hline $\begin{array}{l}\text { GUERRA H. S, et al, } \\
2017\end{array}$ & $\begin{array}{l}\text { Avaliar o risco de } \\
\text { quedas em idosos que } \\
\text { são acompanhados por } \\
\text { uma unidade de saúde } \\
\text { da Atenção Primária } \\
\text { no município de } \\
\text { Aparecida de Goiânia, } \\
\text { GO. }\end{array}$ & $\begin{array}{l}\text { Participaram da } \\
\text { pesquisa } 97 \text { idosos, } \\
\text { sendo a maioria do } \\
\text { sexo feminino. A } \\
\text { idade variou de } 60 \text { a } \\
95 \text { anos. }\end{array}$ & $\begin{array}{l}\text { Foi utilizado o TUG para avaliar o } \\
\text { equilíbrio em idosos e fez uso de } \\
\text { questionários para a coleta dos } \\
\text { dados. }\end{array}$ & $\begin{array}{l}\text { Foi verificado que a maioria dos } \\
\text { idosos demonstraram baixo risco } \\
\text { de quedas, mas foi observado um } \\
\text { terço dos idosos relataram já ter } \\
\text { sofrido algum episódio de quedas. }\end{array}$ \\
\hline $\begin{array}{l}\text { RODRIGUES M. M. P, } \\
\text { et al, } 2017\end{array}$ & $\begin{array}{l}\text { Analisar o risco de } \\
\text { quedas em idosos que } \\
\text { residem em uma } \\
\text { comunidade } \\
\text { nordeste do BR. }\end{array}$ & $\begin{array}{l}\text { Estudo realizado cm } \\
204 \text { idosos de ambos } \\
\text { os sexos. }\end{array}$ & $\begin{array}{l}\text { Utilizou-se questionário } \\
\text { sociodemográfico, analise de } \\
\text { incidência de quedas e utilização d } \\
\text { teste TUG. }\end{array}$ & $\begin{array}{l}\text { O estudo analisou que quanto } \\
\text { maior a idade do idoso, maior a } \\
\text { dependência para locomoção e } \\
\text { maior o risco de quedas. Mas } \\
\text { teste TUG não demostrou eficácia } \\
\text { para prever quedas, quando } \\
\text { utilizado sozinho. }\end{array}$ \\
\hline CHOW R. B et al, 2018 & $\begin{array}{l}\text { Comprovar a eficácia } \\
\text { do teste TUG e do } \\
\text { teste de cadeira para } \\
\text { uso durante a } \\
\text { avaliação de risco de } \\
\text { quedas em idosos em } \\
\text { um departamento de } \\
\text { emergência. }\end{array}$ & $\begin{array}{l}\text { Realizado com } 192 \\
\text { idosos de ambos os } \\
\text { sexos. }\end{array}$ & $\begin{array}{l}\text { Este estudo utilizou o TUG e o teste } \\
\text { da cadeira como forma de avaliação, } \\
\text { além de outros questionários. Todos } \\
\text { os foram } \\
\text { acompanhados por telefone durante } \\
6 \text { meses após o inicio do estudo, } \\
\text { onde os mesmo relatavam quaisquer } \\
\text { quedas que possa ter ocorrido } \\
\text { durante esse período. }\end{array}$ & $\begin{array}{l}\text { Concluindo que os testes não } \\
\text { obtiveram um bom desempenho } \\
\text { como avaliadores de risco de } \\
\text { quedas em idosos no mesmo } \\
\text { departamento. }\end{array}$ \\
\hline $\begin{array}{l}\text { FIORITTO A. P et al, } \\
2020\end{array}$ & $\begin{array}{l}\text { Estimar a prevalência } \\
\text { do risco de quedas e } \\
\text { analisar os fatores } \\
\text { associados a ela em } \\
\text { idosos comunitários } \\
\text { residentes em Juiz de } \\
\text { Fora- MG. }\end{array}$ & $\begin{array}{l}\text { Realizado com } 339 \\
\text { idosos comunitários } \\
\text { com idade de } 60 \\
\text { anos ou mais, de } \\
\text { ambos os sexos. }\end{array}$ & $\begin{array}{l}\text { Este estudo utilizou um questionário } \\
\text { para identificar o perfil } \\
\text { sociodemográfico e questões de } \\
\text { saúde. Além disso, várias escalas } \\
\text { foram utilizadas, entre elas a EFE, } \\
\text { TUG, o TDR, entre outras. O } \\
\text { histórico de quedas foi autorrelatado } \\
\text { e a necessidade de ajuda para andar } \\
\text { também foi avaliada. }\end{array}$ & $\begin{array}{l}\text { Foi evidenciada na pesquisa a alta } \\
\text { prevalência de moderado e } \\
\text { também de alto risco de quedas. }\end{array}$ \\
\hline
\end{tabular}

Legendas: TUG= Timed Up and Go; CE= Ceará; UBS: Unidade Básica de Saúde; $\mathrm{Ba}=$ Bahia; $\mathrm{GO}=\mathrm{Goiás}$; BR= Brasil; $\mathrm{MG}=\mathrm{Minas}$ Gerais; TAF= Teste de Alcance Funcional; EFE= Escala de Fragilidade de Edmonton; TRD= Teste do Relógio; Fonte: Andrade, Costa, Diogenes e Pimentel (2021)

\section{Discussão}

De acordo com envelhecimento o idoso fica mais sujeito a queda, se tratando então de um problema de saúde pública (Guimarães e Farinatti, 2005; Veras, 2009). Embora não seja uma consequência inevitável do envelhecimento, pode sinalizar o início de fragilidade, deixando o equilíbrio comprometido. Além disso, há duas constâncias que sempre estão presente para que isso ocorra como, a perturbação do equilíbrio e deficiência dos sistemas responsáveis pelo controle postural em compensar esta perturbação (Paixão JR \& Heckman, 2006).

No estudo de Bretan et al., 2013, foi utilizado o Timed Up And Go (TUG) para analisar o equilíbrio de 102 idosos com idade igual ou acima de 75 anos, onde $70 \%$ obtiveram pontuação menor que 10 segundos ou entre 10 e 19 segundos. Já os $30 \%$ restantes tiveram pontuação entre 20 e 29 ou 30 ou mais segundos, sendo que a maioria dos idosos apresentaram baixo 
risco de quedas, demonstrando estarem funcionalmente ativos, constatando assim, que o TUG é uma boa ferramenta para avaliar o risco de quedas.

Virtuoso J. F, et al., 2013, na sua pesquisa para analisar versão clássica e cognitiva do TUG na previsão de idosos praticamente de exercício físico observou-se que são vários fatores que influenciam no episódio de quedas em idosos além do nível de atividade física. Porém não foram encontrados estudos que comprovem sua associação em idosos praticantes de exercícios físico. Os testes TUG clássico e cognitivo não se associam com a ocorrência de quedas e devem ser utilizados com cautela para previsão de quedas em idosos praticantes de EF. É necessário algumas variáveis como o tempo de prática de exercícios, população idosa mais ativa, o uso de equipamentos como força plataformas que tornam-se indispensável da prática clínica.

Já na pesquisa de Campos et al., 2013, o estudo foi aplicado em 155 idosos e foram utilizados dois testes funcionais de equilíbrio, porém não demonstraram que eles previnem quedas em idosos, apresentando resultados médios próximos nos grupos queda e não-queda, sem diferença significativa, mesmo quando estratificados por sexo.

No estudo de Rodrigues e Souza 2016, por sua vez, foi utilizado o TUG para avaliar sua eficiência na prevenção de quedas em idosos, onde se observou uma prevalência do sexo feminino, e 16,4\% da amostra informaram que sofreram quedas no último mês. Além disso, a pesquisa identificou com a utilização do teste TUG que 98,2 \% da amostra não possui ou é baixo o risco para quedas, e 1,8\% demostrou risco para quedas significativo. Com isso concluiu-se que o teste TUG demostra eficiência para avaliação de risco de quedas em idosos.

Entretanto, no estudo realizado por Kojima et al., 2015, com 259 idosos acima de 65 anos, que também se analisou a capacidade do TUG para prováveis quedas, houve um resultado não favorável à eficiência do teste. O referido estudo frisa que as quedas são comuns nos idosos, e utilizam o TUG como uma triagem para verificar os riscos de quedas, no entanto, o mesmo é limitado e, é indicado para alto índice de quedas nos idosos, podendo-se dizer que este estudo se trata de um corte ideal. Os autores consideram, ainda, que o ponto fora deste estudo tem baixa sensibilidade, alta especificidade e alto valor preditivo negativo, indicando, dessa forma, que apesar do tempo, o TUG pode não ser aditivo ao histórico de quedas e na identificação delas, exigindo diagnósticos precisos, avaliação e intervenção, para ajudar a excluir aqueles de baixo risco.

Assim como em outros dois estudos que foram analisados, foi possível observar a predominância do sexo feminino e que a maioria dos idosos já sofreu pelo menos uma queda no último ano. Os estudos também corroboram com o pensamento que quanto maior a idade maior o risco de sofrer quedas. As pesquisas puderam perceber que o teste TUG não mostrou eficiência na predição de quedas em idosos e propõem que se tenha mais pesquisas com a utilização do TUG para demostrar sua eficácia. Além da necessidade de atenção com esse tema, pois a queda é um acontecimento que acomete muitos idosos, é muito importante se avaliar o risco de quedas para se tomar as medidas necessárias. (Guerra, et al., 2017; Rodrigues, et al., 2017).

Já na pesquisa de Martinez et al., 2016, onde foi utilizado o teste TUG para avaliar a segurança e reprodutibilidade do teste em idosos hospitalizados, a média no valor de corte foi de 10,0 o que está aproximado do valor normal na população brasileira, mas não pode ser apontado como valor de referência para quedas, pois, há estudos brasileiros atuais que verificaram um valor de corte de TUG para quedas em 12,47 segundos, que seria um número maior do que o valor médio deste estudo. Embora o valor médio de TUG encontrar-se entre a faixa de normalidade, por volta de um terço dos idosos hospitalizados revelaram já ter sofrido pelo menos uma queda no período de um ano, o que indica algum tipo de déficit durante execução comparação do desempenho físico. A pesquisa concluiu que o teste TUG foi classificado como um instrumento seguro e de boa reprodutibilidade, mas é preciso realizar três medidas para que o corte de TUG não seja desconsiderado entre os idosos.

Observamos, porém, que os estudos de CHOW et al. 2018, que utilizou o teste TUG e o teste de cadeira com um universo amostral de composta por 192 senescentes de ambos os sexos, corroborou com a pesquisa de Martinez et al (2016), 
onde a maioria dos participantes era do sexo feminino, e também 26,6\% relataram ter sofrido alguma queda nos últimos seis meses. O valor preditivo positivo de TUG foi de $26,3 \%$. O mesmo concluiu que a eficácia do teste TUG e do teste de cadeira tem limitado a capacidade preditiva para risco de quedas. Os pesquisadores consideraram ter uma ferramenta melhor de avaliação de risco de quedas, sendo válido também perguntar se o paciente caiu no último ano. Além disso, foi questionado a variabilidade de valores de corte, já que outros estudos utilizam valores de cortes diferentes. Por fim, os pesquisadores aconselham um aprofundamento do estudo para incluir o uso de outra pontuação ou outra ferramenta para avaliação de futuras quedas.

Seguindo esse raciocínio no estudo realizado por Fioritto et al., 2020, que teve a finalidade de calcular o predomínio de quedas, com a utilização do Timed up and go como ferramenta de avaliação e os fatores relacionados a queda como: ansiedade, depressão, medo de cair, entre outros, avaliados pelo Patient Health Questionnaire, Falls Efficacy ScaleInternacional. Esta pesquisa feita com uma amostra de idosos 339 idosos com 60 anos ou mais de ambos os sexos. Os valores de referências utilizados para a classificação dos indivíduos foram: baixo para 10 segundos ou menos, moderado para o tempo entre 11 e 20 segundos e alto risco de queda para o tempo de realização do teste maior que 20 segundos. Dentre os idosos avaliados pelo TUG, 36 que precisaram de ajuda para se locomover tiveram resistência ou foram incapazes de realizar o teste. Os demais apresentaram uma porcentagem de $36 \%$ para baixo risco de queda, $43,7 \%$ para risco de queda moderado e $20,3 \%$ para alto risco de queda, concluindo que houve grande predomínio de moderado e alto risco de queda. Neste estudo o TUG foi considerado um como um instrumento de grande importância para a saúde pública, pois possibilita a identificação da população de risco.

\section{Conclusão}

Por tanto a partir dos estudos apresentados os achados citados no referido estudo, não demostraram a eficiência do teste TUG para predição do risco de quedas em idosos, mas as pesquisas demostram que o teste tem resultados positivos quando complementado a outro teste. Nota-se a necessidade de escalas eficazes que irão contribuir para a prática clínica e que possam avaliar o risco de quedas pois esse conhecimento é fundamental para a criação de políticas públicas de atenção à saúde direcionadas a esse grupo e assim orientar efetivamente o fisioterapeuta a realizar as avaliações.

Observa-se a escassez de artigos é a necessidade de mais estudos para identificar a veracidade do teste assim como uma medida de corte única, pois existem várias divergências em relação ao valor ideal. E após toda a análise e informações obtidas das outras pesquisas já realizadas sobre o tema, ressaltamos que se pode recomendar o uso do TUG para compor o diagnóstico de mobilidade funcional, e equilíbrio em idosos.

\section{Agradecimentos}

Agradecemos imensamente à Deus por ter permitido que nós tivéssemos saúde e determinação para não desanimar durante a realização desse trabalho.

\section{Referências}

Alexandre, T. S. et al. (2012). Accuracy of Timed Up and Go Test for screening risk of falls among community-dwelling elderly. Rev Bras Fisioter. 16(5), $381-8$.

Baixinho, C. L. et al. (2019). Falls in institutionalized elderly with and without cognitive decline A study of some factors. Dementia \& Neuropsychologia. 13 (1), 116-121.

Bischoff, H.A. et al. (2003). Identifying a cut-off point for normal mobility: A comparison of the timed 'up and go' test in community-dwelling and institutionalised elderly women. Age Ageing. 315-320. 
Bretan, O. et al. (2013). Risk of falling among elderly persons living in the community: assessment by the Timed up and go test. Braz J Otorhinolaryngol. 79(1), 18-21.

Campos, M. P. S. et al. (2013). Os testes de Equilíbrio, Alcance Funcional e “Timed Up and Go" e o risco de queda em idosos. Revista Kairós Gerontologia. $16(4), 125-138$.

Chow, R. B. et al. (2018). Effectiveness of the "timed up and go" (TUG) and the chair test as screening tools for geriatric fall risk assessment in the ED. The American Journal of Emergeney Medicine.

Cunha, J. S., et al. (2019). Produção Científica Acerca da Assistência de Enfermagem no Envelhecimento Saudável da Pessoa Idosa. Faculdade de Enfermagem São Vicente de Paula. 159-176.

Damián, J. et al. (2013). Factors associated with falls among older adults living in institutions. BMC Geriatr. 15, 13-6.

Fang X. et al. (2012). Frailty in relation to the risk of falls, fractures, and mortality in older Chinese adults: results from the Beijing Longitudinal Study of Aging. The Journal of Nutrition, Health \& Aging, 16(10), 903-907.

Fioritto, A.P. et al. (2020). Prevalência do risco de queda e fatores associados em idosos residentes na comunidade. Rev. Bras. Geriatr. Gerontol. 23(2), 200076

Gamage, N. et al. (2019). Prevalence and associated risk factors of falls among rural community-dwelling older people: a cross- sectional study from southern Sri Lanka. Current Gerontology and Geriatrics Research.

Guerra, H. S. et al. (2017). Avaliação do risco de quedas em idosos da comunidade. Rev. Saúde.Com. 13(2), 879-886

Guimarães, J. M. N, \& Farinatti, P. T. V. (2005). Análise descritiva de variáveis teoricamente associadas ao risco de quedas em mulheres idosas. Rev Bras Med Esporte. 11, 299-305.

Jagnoor J. et al. (2014). A qualitative study on the perceptions of preventing falls as a health priority among older people in. Northern India. Inj Prev. 20, 2934 .

Kojima, et al. (2015). Does the timed up and go test predict futurefalls among British community-dwelling older people? Prospective cohort study nested within a randomised controlled trial. BMC Geriatrics. 15:38.

Lima-Costa, M. F. (2018) Envelhecimento e saúde coletiva: Estudo Longitudinal da Saúde dos Idosos Brasileiros (ELSI-Brasil). Rev Saude Publica. 52, 2-2.

Martinez, B. P. et al. (2016). Segurança e reprodutibilidade do Teste Timed Up and Go em idosos hospitalizados. Rev Bras Med Esporte. 22(5).

Paixão, J. C. M., \& Heckman, M. F. (2006). Distúrbios da postura, marcha e quedas. In: Freitas, E.V. et al. Tratado de Geriatria e Gerontologia. Guanabara Koogan, 950- 959.

Podsiadlo, D., \& Richardson, S. (1991). The timed "Up \& Go": a test of basic functional mobility for frail elderly persons. Journal of the. American Geriatrics Society. 39(2), 142-148.

Rodrigues, et al. (2017). Timed Up and Go Risk Predictor of Falls in Elderly People Residing in the Community? International Archives of Medicine. Vol. 10(146).

Rodrigues, A. L. P., \& Souza, V. R. (2016). Eficiência do Teste Timed Up and Go na predição de quedas em idosos atendidos em uma Unidade Básica de Saúde de Fortaleza-Ce. Revista Brasileira de Prescrição e Fisiologia do Exercício. 10(58), 314-320.

Rodrigues, I. G et al. (2014). Falls among the elderly: risk factors in a population-based study. Rev. Brasileira de Epidemiologia. 17(3), 705-718.

Rother, E. T. (2017). Revisão sistemática X revisão narrativa. Acta Paulista de Enfermagem. 20(2), 5-6.

Silva, S. M. C. S., \& Mura, J. D. P. (2014). Tratado de alimentação, nutrição e dietoterapia. (2a ed.), Roca. $461-462$.

Smith, A. A. et al. (2017). Assessment of risk of falls in elderly living at home. Rev. Latino- Am. Enfermagem. $25,2754$.

Sociedade Brasileira de Geriatria e Gerontologia (2019). OMS divulga metas para 2019; desafios impactam a vida de idosos.

Teixeira, D. K. S. et al. (2019). Falls among the elderly: environmental limitations and functional losses. Revista Brasileira de Geriatria e Gerontologia. 22(3), $1-10$

Teresi, J. A. et al. (2013). Comparative effectiveness of implementing evidence-based education and best practices in nursing homes: Effects on falls, qualityof-life and societal costs. International Journal of Nursing Studies. 50(4), 448-463.

Thrane, G. et al. (2007). Thornquist E. The association between timed up and go test and history of falls: The Troms $\emptyset$ study. BMC Geriatr. 7(1),1-7.

Veras, R. (2009). Envelhecimento populacional contemporâneo: demandas, desafios e inovações. Rev Saúde Pública. 43, 548-554.

Virtuoso, J. F. et al. (2013). Revista Brasileira de Cineantropometria e Desempenho humano. Rev Bras Cineantropom Desempenho Hum. 16(4), 381-389. 